\title{
Retzius-sparing robot-assisted laparoscopic radical prostatectomy: functional and early oncologic results in aggressive and locally advanced prostate cancer
}

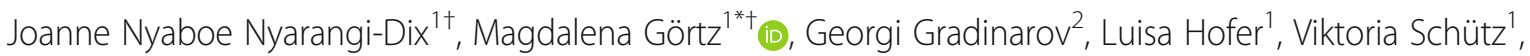
Claudia Gasch', Jan Philipp Radtke ${ }^{1}$ and Markus Hohenfellner ${ }^{1}$

\begin{abstract}
Background: Retzius-sparing robot-assisted laparoscopic radical prostatectomy (rsRARP) allows entire prostatectomy procedure via the pouch of Douglas. In low- and intermediate-risk prostate cancer (PCa) there is level 1 evidence that the Retzius-sparing approach impacts early continence recovery. Since specific data on aggressive and locally advanced cancer is lacking and avoiding rsRARP is presently suggested, we investigated urinary and sexual recovery, perioperative complications and early oncologic outcomes after rsRARP in this particular cohort.

Methods: Prospectively collected data of 50 consecutive men (median age 66 years) with high-risk PCa who underwent rsRARP in a single institution was analysed retrospectively. The follow-up for all patients was 12 months after surgery.

Results: 3 vs. 12 months after surgery, $82 \%$ vs. 98\% of men used no pad or one safety pad and 50\% vs. $72 \%$ used no pad. $89 \%$ of patients did not observe a decline of continence if postoperative radiotherapy was carried out. Considering the 17 preoperatively potent patients who underwent bi- or unilateral nerve-sparing surgery, 41\% reported their first sexual intercourse within 1 year after rsRARP.

$84 \%$ of patients had $\geq \mathrm{p} T 3$ a disease and $42 \%$ positive surgical margins. A lymphadenectomy was done in $94 \%$ of patients with a median lymph node removal of 15 and lymph node metastasis in 13\%.

34\% underwent adjuvant radiotherapy and 22\% adjuvant androgen deprivation therapy (ADT). 1-year recurrence-free survival was $96 \%$, including $25 \%$ of patients on adjuvant or salvage ADT.

Conclusions: RsRARP in high-risk PCa is feasible and results in excellent continence rates, even after postoperative radiotherapy. The potency rates are promising but need further clarification in larger cohorts. Reliable oncologic outcomes require longterm follow-up and are awaited.
\end{abstract}

Keywords: High risk prostate cancer, Retzius-sparing robot-assisted radical prostatectomy, Urinary continence, Erectile function

\footnotetext{
* Correspondence: magdalena.goertz@med.uni-heidelberg.de

†Joanne Nyaboe Nyarangi-Dix and Magdalena Görtz contributed equally to

this work.

${ }^{1}$ Department of Urology, University Hospital Heidelberg, Im Neuenheimer

Feld 110, 69120 Heidelberg, Germany

Full list of author information is available at the end of the article
}

(c) The Author(s). 2019 Open Access This article is distributed under the terms of the Creative Commons Attribution 4.0 International License (http://creativecommons.org/licenses/by/4.0/), which permits unrestricted use, distribution, and reproduction in any medium, provided you give appropriate credit to the original author(s) and the source, provide a link to the Creative Commons license, and indicate if changes were made. The Creative Commons Public Domain Dedication waiver (http://creativecommons.org/publicdomain/zero/1.0/) applies to the data made available in this article, unless otherwise stated. 


\section{Background}

After Binder and Kramer [1] reported the first robotassisted laparoscopic radical prostatectomy (RARP) in 2001, RARP proceeded to become a widespread and accepted surgical approach for prostatectomy [2]. Meanwhile, the "holy grail" in routine RARP consists of a complete tumor resection and optimal functional results [3]. With oncological outcomes after RARP in localized prostate cancer ( $\mathrm{PCa}$ ) being steadfast, focus is currently shifting towards improvement of perioperative morbidity and functional results.

Reports suggest that surgical techniques involving membranous urethral length preservation [4], posterior musculofascial reconstruction [5] and/or bladder neck preservation [6] may further improve continence. In 2010, Bocciardi et al. reported an innovative posterior Retzius-sparing RARP (rsRARP) approach avoiding bladder detachment [7] thereby presumably minimizing surgical trauma and preserving normal pelvic anatomy maximally. The continence rate reported within the first year of surgery was 96\% [8]. In another prospective study, $71 \%$ of men after rsRARP were continent 1 week after catheter removal vs. $48 \%$ after conventional RARP [9]. These promising results were reported in studies that entirely focused on low- or intermediate-risk PCa collectives. Unknown for this novel technique is whether these functional outcomes can be replicated alongside good oncological outcomes in the challenging setting of high-risk and locally advanced PCa [10].

Men with PCa and a prostate-specific antigen (PSA) > $20 \mathrm{ng} / \mathrm{ml}$, Gleason score $8-10$ or clinical-stage $\geq \mathrm{T} 3$ are recognized by the major international guidelines to be advanced $\mathrm{PCa}$ and constitute the high-risk PCa group [11]. This advanced high-risk-group warrants RP with a clear need for radical/wider dissection, which may compromise functional outcomes [12]. Concerns in these high-risk tumors also regard positive surgical margins (PSMs), inadequate disease control and increased side effects [13]. Recently, accompanied by advances in surgical technology, $\mathrm{RP}$ alone or as an initial step within a multimodal concept has gained acceptance in locally advanced PCa [14]. Men with locally advanced PCa treated with RP plus radiotherapy have a lower risk of prostate cancer-specific death compared to men treated with radiotherapy plus androgen deprivation therapy (ADT) alone [15]. EAU guidelines now recognize the role of RP in men with locally advanced $\mathrm{PCa}$, if required in combination with additional treatments such as radiotherapy or ADT [11].

Since rsRARP promotes early continence recovery without an impact on biochemical failure in low- and intermediate-risk $\mathrm{PCa}$, we investigated rsRARP in highrisk PCa. We report the functional, early and midterm oncologic results of our first 50 RARP-patients with high-risk PCa treated by the Retzius-sparing approach.

\section{Methods}

\section{Patient cohort}

This study was a retrospective review of prospectively collected data at the Department of Urology at Heidelberg University Hospital. The study was approved by the ethical committee of the University of Heidelberg (S403/2012). Our analytic cohort included 50 consecutive patients with aggressive and/or locally advanced $\mathrm{PCa}$ undergoing routine rsRARP between July 2016 and September 2017. Men were classified as high-risk when PSA level $>20 \mathrm{ng} / \mathrm{ml}$, Gleason score $8-10$ and/or a pathological stage $\geq \mathrm{T} 3$ was met, according to recent EAU guidelines [11].

\section{Surgical technique}

All prostatectomies were performed by 3 experienced surgeons routinely performing over 100 RARPs per year. Our rsRARP technique was largely similar to that first described by Galfano et al. [7]. Modifications used in our technique are as described below:

A standard, four-arm da Vinci Si surgical robot was used. Surgeons began with a $0^{\circ}$ surgical lens and mostly switched to a $30^{\circ}$-upwards lens beginning at the preparation of the bladder neck henceforth. As a slight technical modification, in contrast to Galfano et al. [7, 8], there was no use of two or four cardinal stitches into the bladder for the identification of the bladder neck orifice during the urethrovesical anastomosis.

\section{Evaluated clinical and oncological parameters}

For each patient, we collected the following clinical and pathologic information: age, preoperative total PSA level, prostate volume, preoperative International Prostate Symptom Score (IPSS), International Index of Erectile Function-5 (IIEF-5) score, biopsy Gleason score, clinical cancer stage (cTNM), prostatectomy Gleason score, pathological cancer stage (pTNM) and PSM rate. Furthermore, the following perioperative variables were evaluated: perioperative transfusion rate, bilateral or unilateral nerve-sparing, intraoperative status of the vesicourethral anastomosis, intraoperative and early postoperative complications, length of hospital stay and time to catheter removal.

Postoperative complications were recorded using the Clavien-Dindo classification [16].

\section{Follow-up}

All patients completed a questionnaire the day before surgery, including the IPSS and the IIEF-5 questionnaire. During the first year after surgery an institutional questionnaire was sent by mail to inquire postoperative complications, the PSA level and the continence and potency recovery. Additionally, patients were interviewed by telephone to 
complete outstanding information. Minimum follow-up was 12 months.

Patients were considered continent when they used $\leq 1$ safety $\mathrm{pad} /$ day. The date on which the patient stopped using more than 1 safety pad was considered the recovery time of continence. Time to recovery of erectile function in preoperatively potent men (IIEF- 5 score > 17) was considered the date of the first sexual intercourse after surgery. All men with PSA $\geq 0.2 \mathrm{ng} / \mathrm{ml}$ at analysis were considered biochemically recurrent [11].

\section{Statistical analysis}

Median and interquartile ranges (IQRs) were used to report nonparametric continuous variables. Continence recovery was demonstrated using a Kaplan-Meier plot (IBM SPSS Statistics 24).

\section{Results}

\section{Perioperative results}

Demographic data are summarized in Additional file 1. The median age was 66 years and the median hospitalization was 4 days. The urethrovesical anastomosis was intraoperatively watertight in $92 \%$ of patients. A cystogram was performed in all cases on postoperative day 10 to 14. In case of extravasation, a new cystogram was planned. The Foley catheter was removed at a median of 12 days (IQR: $10-14$ days).

Complications were classified according to the Clavien-Dindo system. Clavien-Dindo grade I, II and III complications took place in 2, 2 and $6 \%$ of cases, respectively. None of the cases required conversion to open surgery. Intraoperative or postoperative blood transfusions were not needed. There was 1 case of rectal perforation which was detected and sutured intraoperatively. Peripheral pulmonary embolism occured in 1 patient. Owed to anastomotic-insufficiency, 1 man was catheterised for 2 months. There were 3 cases of symptomatic lymphoceles, 2 of them requiring percutaneous drainage (Clavien-Dindo grade IIIa) and one requiring laparoscopic lymphocele-marsupialization (ClavienDindo grade IIIb).

\section{Oncologic results}

Postoperatively, pathological evaluation revealed 32\% with a Gleason score of $\geq 8$ and $84 \%$ with extraprostatic extension (pT3-disease). The overall PSM rate was $42 \%$ (21 of 50 patients). Eleven patients were identified to have an anterior index tumor in imaging. 8 of 11 (73\%) patients with anterior tumors had pT3-disease and 5 of 11 (45\%) had PSM. A lymphadenectomy was done in $94 \%$ of patients with a median lymph node removal of 15 (IQR 10-21). Lymph node metastasis was documented in 6 of 47 patients (13\%).
Postoperative PSA persistence (defined as a PSA level $\geq$ $0.2 \mathrm{ng} / \mathrm{ml}$ at 3 months after surgery) was documented in 5 of $48(10 \%)$ patients. $34 \%$ of patients underwent adjuvant radiotherapy and $22 \%$ of patients adjuvant ADT.

During 1-year follow-up 2 (4\%) patients experienced biochemical recurrence. 2 (4\%) patients received salvage treatments: radiotherapy alone or radiotherapy plus ADT. 1-year biochemical disease-free survival with a PSA $\leq 0.2 \mathrm{ng} / \mathrm{ml}$ was $96 \%$. Among them, 12 (25\%) patients were on ADT.

\section{Continence results}

The urinary continence rate was $38 \%$ at 1 week after catheter removal. Three months after surgery, $82 \%$ were continent. Figure 1 shows the recovery curve for continence.

Twelve months postoperatively, 98\% were continent: while $73 \%$ used no pad, $27 \%$ used one safety pad per day. One man (2\%) used two pads per day. 17 of 19 (89\%) who had received adjuvant or salvage radiotherapy observed no decline of continence after radiotherapy. To date, no anastomotic stricture has been detected.

\section{Erectile function results}

Detailed information about sexual function recovery is shown in Additional file 2. 29 (58\%) patients were preoperatively potent with an IIEF- 5 score $>17$. In those patients, bilateral and unilateral nerve-sparing was performed in $12(41 \%)$ and $5(17 \%)$ men, respectively. 7 of the 17 (41\%) who underwent bilateral or unilateral nervesparing surgery reported to have regular and consistent sexual intercourse within the first year of surgery. 2 of the 7 (29\%) postoperatively potent patients used phosphodiesterase type 5 inhibitors on-demand. Median time to recovery of erectile function was 8 months (IQR: 6-11 months). For the 7 postoperatively potent patients, the median IIEF frequency score (Q3) to maintain the erection during sexual intercourse was 4 (IQR: $3-4)$ and the median IIEF satisfaction score (Q5) was 4 (IQR: 4-5).

\section{Discussion}

Since the Bocciardi group described their technique of a Retzius-sparing approach of RARP in 2010, excellent functional and oncological outcomes have been reported $[7,8]$. A recent review found that rsRARP is associated with earlier continence recovery compared to conventional RARP without significant impact on oncological outcomes [17]. Beyond the initiator group, subsequent studies focused on low- and intermediate-risk PCa, leaving a gap in reporting for the high-risk and locally advanced disease cohort [9]. Consequently, due to the lack of data on the outcomes in higher-risk $\mathrm{PCa}$, it has been advised to limit the Retzius-sparing approach to low- or favorable intermediate-risk disease [18]. Since adapting Retzius-sparing prostatectomy in our centre, all men 


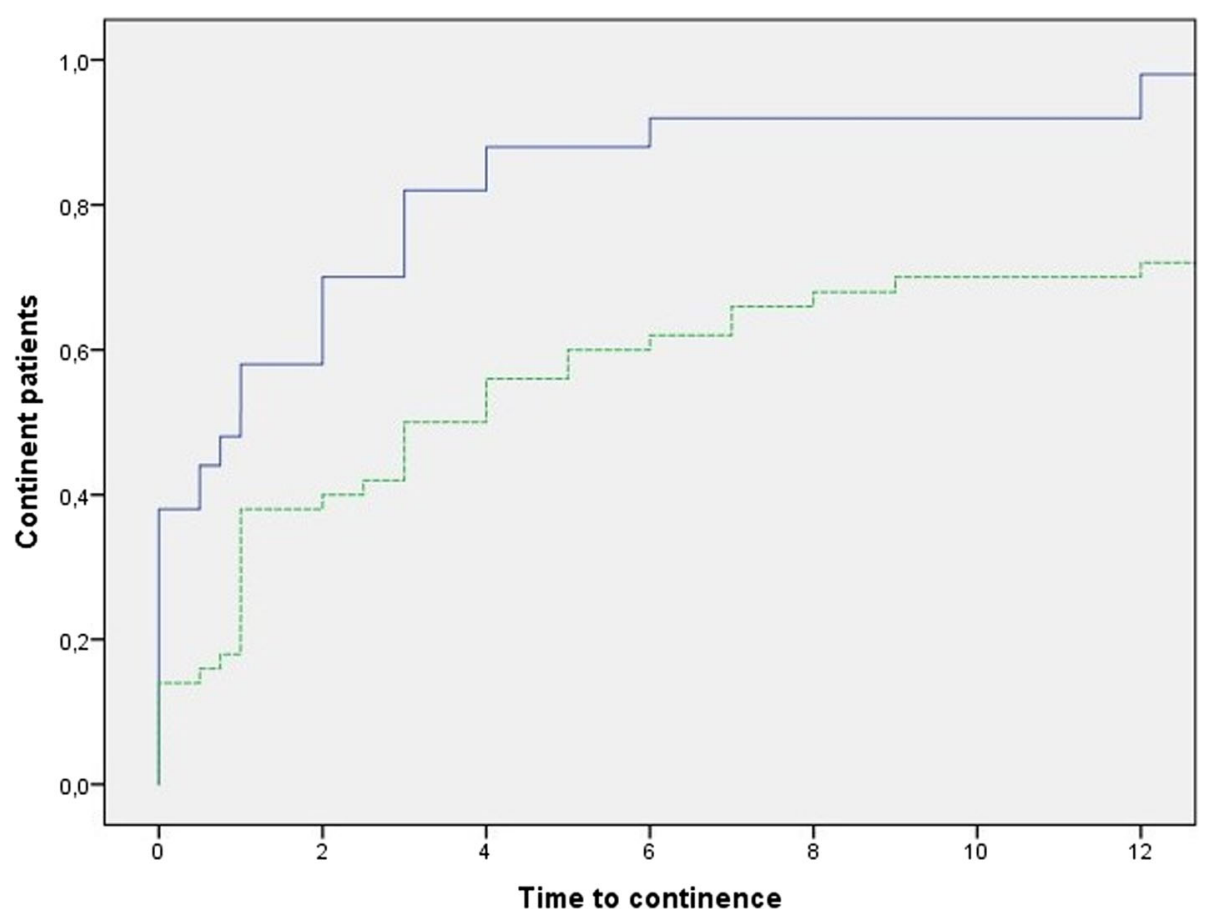

Fig. 1 Kaplan-Meier curve of continence recovery. Blue continuous line = no pad or one safety pad; green dashed line = no pad. Time to continence is expressed in months

viable for RARP were operated in this manner, regardless of preoperative oncological characteristics. We hereby present the first report that focuses on the perioperative, functional and oncologic results after rsRARP in a small purely aggressive PCa-collective.

Our perioperative results show that Retzius-sparing prostatectomy is not only feasible but safe in aggressive and locally advanced PCa. According to the ClavienDindo classification, only Grade III complications or less were detected. Grade III complications occurred in 3 (6\%) patients and were due to extended lymphadenectomy yielding up to 40 lymph nodes per patient. Lymphadenectomy is mandatory in this cohort, since it improves the accuracy of cancer staging [19] and may be oncologically beneficial [20]. The lymphocele rate in our study was in accordance with another series reporting occurance in $10 \%$ of patients undergoing extended pelvic lymph node dissection [21].

As would be expected in an aggressive and locally advanced collective with PSA level $>20 \mathrm{ng} / \mathrm{ml}$, Gleason score $>7$ and/or pathological stage $\geq \mathrm{T} 3$, PSMs were higher than in a normal/mixed cohort. In the present analysis, $84 \%$ of patients harbored extraprostatic $\geq \mathrm{pT} 3 \mathrm{a}$ disease. The present PSM rate (42\%) is within range of other reports including a review on high-risk $\mathrm{PCa}$ treated with conventional RARP that reported a $16-58 \%$ PSM rate [22]. Harty and colleagues performed conventional RARP in a cohort more similar to the present study (high risk and locally advanced $\mathrm{PCa}$ ). In their cohort with $78 \% \geq$ pT3a disease, $50 \%$ had PSMs [23]. An explanation for a reduced PSM rate in rsRARP may be the possibility to dissect antegradely allowing improved visualization of the prostate base and posterior margin of the prostate, which are common sites of PSMs in conventional RARP [24].

Despite knowledge of higher likelihood of PSMs and biochemical recurrence in locally advanced $\mathrm{PCa}$, studies have shown overall survival rates of $>75 \%$ at 10 years after RP [22]. Adjuvant therapies following prostatectomy play an important role in surviving locally advanced and high-risk PCa. Reduced biochemical recurrence, distant metastases and longer survival rates of high-risk patients have been repeatedly proved after adjuvant radiotherapy [25]. The role of hormonal manipulation therapy in the high-risk collective is embedded in all national and international guidelines [11]. In our cohort, 34 and $22 \%$ of patients underwent adjuvant radiotherapy and adjuvant $\mathrm{ADT}$, respectively. Adjuvant radiotherapy was initiated in men at a high risk of progression according to the histopathological results, e.g. extracapsular extension and positive margins in final pathology specimen results. Adjuvant ADT was initiated in men with lymph node metastasis or with very highrisk localized disease. Twelve months after surgery, $4 \%$ of men had received salvage radiotherapy and $2 \%$ salvage ADT. 
1-year progression-free survival in our cohort was $96 \%$; this included $12(25 \%)$ patients on ADT. In a previously published study on patients with high-risk PCa treated by conventional RARP, the 1-year biochemical recurrence-free survival was $81 \%$, with only $6 \%$ primarily scheduled to receive adjuvant treatment [26]. However, current data support the view that overall progressionfree survival of high-risk patients is significantly increased when planned adjuvant therapy is administered [25]. It is clear though, that longer oncological follow-up must be awaited.

Defining urinary continence as maximum use of a safety pad per day, $98 \%$ of men were continent 12 months after rsRARP in this high-risk cohort. $72 \%$ of men were pad free 12 months after rsRARP. Studies of patients with high risk or locally advanced prostate cancer show heterogeneous continence outcomes after RARP. Abdollah et al. analyzed the urinary function of 769 men with high-risk PCa treated with conventional RARP at two high-volume academic centers and revealed urinary continence recovery in $85 \%$ of men at 12 months after surgery (defined as the use of no pad or one safety pad per day) [12]. Gandaglia et al. found a 1year urinary continence recovery rate of $64 \%$ after conventional RARP in locally advanced cancer (defined as the use of no pad) [27].

Regarding early continence recovery, $38 \%$ of men in our study were continent 1 week after catheter removal and $82 \%$ of men 3 months after surgery. With rsRARP in low- and intermediate-risk $\mathrm{PCa}$, early continence was reported in $71 \%$ of men 1 week after catheter removal and in $95 \%$ of men 3 months after surgery [9]. One explanation for this discrepancy is that our cohort included locally advanced disease with $84 \%$ harboring extraprostatic extension in contrast to the previous predominantly pT2-cohort study $[9,28]$. The oncological necessity of a more radical and wider dissection plane may explain generally reduced continence rates in high-risk collectives [12]. At the same time, prolonged postoperative catheterisation in the present cohort may have played a vital role. Furthermore, the proneness of high-risk cancer patients to delayed recovery of urinary continence after prostatectomy may also be due to the broader lymphadenectomy template necessary in high-risk $\mathrm{PCa}$; increasing possible damage to nerve fibers innervating the pelvic floor [29]. Nevertheless, Retzius-sparing prostatectomy appears to substantially improve continence rates, rendering a great proportion $(84 \%)$ of this highrisk cohort, ready for further multimodal therapies like adjuvant radiotherapy.

Men receiving postoperative radiotherapy are known to have a higher overall incontinence rate [30]. Interestingly, only 2 of 19 (11\%) patients who received radiotherapy after rsRARP observed a decline in continence after radiotherapy. Previous studies revealed that only $22 \%$ of men who are continent at the start of radiotherapy remained continent afterwards [31]. During rsRARP most attachments of the bladder remain untouched and pubourethral and pubovesical attachments remain intact avoiding bladder/urethral hypermobility [32]. This may have a protective effect for continence after radiotherapy. Considering this impact for the adjuvant radiotherapy prone high-risk PCa-group, the Retzius-sparing technique should be investigated in further studies.

For oncological reasons, preservation of erectile function is not highly propagated in men confronted with the diagnosis of high-risk disease [33]. Nonetheless, of the preoperatively potent men with a strong wish for neurovascular bundle preservation and an IIEF-5 score > 17 who underwent unilateral or bilateral nerve-sparing, $41 \%$ had had sexual intercourse in the year following rsRARP. Abdollah et al. performed nerve-sparing in $88 \%$ of patients with high-risk $\mathrm{PCa}$ undergoing conventional RARP and reported that $26 \%$ of preoperatively potent men were able to have intercourse 12 months after surgery [12].

Since recovery of sexual function in general continues beyond 12-24 months post surgery [12], it is too early to estimate the full percentage of men that can expect to recover sexual function after rsRARP in high-risk PCa. Neurovascular bundle preservation should, nonetheless, be reserved to highly informed men consenting to nerve-sparing despite increased risk of PSMs in aggressive and locally advanced PCa.

A limitation of this study was that it is nonrandomized, non-comparative and retrospective in nature. However, this represents a natural step during the exploration of a new surgical technique. In light of the results of the current study, we initiated a prospective randomized clinical trial at our institution that compares the outcomes of conventional and rsRARP in low-, intermediate and high-risk PCa.

Another limitation was that a single, highly experienced team performed RARP at an academic institution and that the results may not be generalizable.

In addition, it is premature to foresee long-term oncological outcomes in this cohort, since results regarding oncologic efficacy and probability of biochemical failure $>12$ months after RARP are pending.

Lastly, the promising first results of sexual function recovery after rsRARP in a purely aggressive PCa-collective need to be confirmed in larger cohorts and over a longer period of time.

\section{Conclusions}

With the increasing role of RARP in the management of aggressive and locally advanced PCa, we present the 1year functional and oncological outcomes in a cohort of 
50 men with high-risk PCa treated with rsRARP. RsRARP is feasible, safe and associated with few perioperative complications. We demonstrate (very) good results regarding the recovery of urinary continence in patients with high-risk $\mathrm{PCa}$. Continence results following rsRARP and postoperative radiotherapy are promising. Potency rates appear encouraging but need further clarification in larger cohorts. Longterm oncological outcomes are awaited.

\section{Supplementary information}

Supplementary information accompanies this paper at https://doi.org/10 1186/s12894-019-0550-9.

Additional file 1. Demographic, preoperative and pathologic features.

Additional file 2. Sexual function recovery.

\section{Abbreviations}

ADT: Androgen deprivation therapy; EAU: European Association of Urology; IIEF-5 : International Index of Erectile Function-5; IPSS: International Prostate Symptom Score; IQR: Interquartile range; PCa: Prostate cancer; PSA: Prostatespecific antigen; PSM: Positive surgical margin; RARP: Robot-assisted laparoscopic radical prostatectomy; RP: Radical prostatectomy; rsRARP: Retzius-sparing robot-assisted laparoscopic radical prostatectomy

\section{Acknowledgements}

Not applicable.

\section{Authors' contributions}

JN: Protocol development, Data analysis, Manuscript writing. MG: Protocol development, Data collection, Data analysis, Manuscript writing. GG: Data collection. LH: Data collection, Manuscript editing. VS: Data collection, Manuscript editing. CG: Manuscript editing. JR: Manuscript editing. MH: Protocol development, Manuscript editing. All authors read and approved the final manuscript.

\section{Funding}

Not applicable.

\section{Availability of data and materials}

The datasets used and analysed during the current study are available from the corresponding author on reasonable request.

\section{Ethics approval and consent to participate}

All procedures performed in studies involving human participants were in accordance with the ethical standards of the institutional and/or national research committee and with the 1964 Helsinki declaration and its later amendments or comparable ethical standards. The study was approved by the ethical committee of the University of Heidelberg (S-403/2012). The consent obtained from study participants was in written form.

\section{Consent for publication}

Not applicable.

\section{Competing interests}

JR has worked as a company consultant for Siemens Healthineers, Bender Group, Beckelmann, Saegeling Medizintechnik, Invivo, Philips and MedCom; none of these sources had any input into this article. The other authors have nothing to disclose.

The authors declare that they have no conflict of interest.

\section{Author details}

${ }^{1}$ Department of Urology, University Hospital Heidelberg, Im Neuenheimer Feld 110, 69120 Heidelberg, Germany. ${ }^{2}$ Ruprecht-Karls University of Heidelberg, Medical Faculty, Heidelberg, Germany.
Received: 1 March 2019 Accepted: 31 October 2019

Published online: 12 November 2019

\section{References}

1. Binder J, Kramer W. Robotically-assisted laparoscopic radical prostatectomy. BJU Int. 2001:87(4):408-10

2. Yaxley JW, Coughlin GD, Chambers SK, Occhipinti S, Samaratunga H, Zajdlewicz L, et al. Robot-assisted laparoscopic prostatectomy versus open radical retropubic prostatectomy: early outcomes from a randomised controlled phase 3 study. Lancet. 2016;388(10049):1057-66

3. Bianco FJ Jr, Scardino PT, Eastham JA. Radical prostatectomy: long-term cancer control and recovery of sexual and urinary function ("trifecta"). Urology. 2005:66(5 Suppl):83-94.

4. Paparel P, Akin O, Sandhu JS, Otero JR, Serio AM, Scardino PT, et al. Recovery of urinary continence after radical prostatectomy: association with urethral length and urethral fibrosis measured by preoperative and postoperative endorectal magnetic resonance imaging. Eur Urol. 2009;55(3): 629-37.

5. Rocco B, Cozzi G, Spinelli MG, Coelho RF, Patel VR, Tewari A, et al. Posterior musculofascial reconstruction after radical prostatectomy: a systematic review of the literature. Eur Urol. 2012;62(5):779-90.

6. Nyarangi-Dix JN, Tichy D, Hatiboglu G, Pahernik S, Tosev G, Hohenfellner M. Complete bladder neck preservation promotes long-term postprostatectomy continence without compromising midterm oncological outcome: analysis of a randomised controlled cohort. World J Urol. 2018; 36(3):349-55.

7. Galfano A, Ascione A, Grimaldi S, Petralia G, Strada E, Bocciardi AM. A new anatomic approach for robot-assisted laparoscopic prostatectomy: a feasibility study for completely intrafascial surgery. Eur Urol. 2010;58(3):457-61.

8. Galfano A, Di Trapani D, Sozzi F, Strada E, Petralia G, Bramerio M, et al, Beyond the learning curve of the Retzius-sparing approach for robotassisted laparoscopic radical prostatectomy: oncologic and functional results of the first 200 patients with $>/=1$ year of follow-up. Eur Urol. 2013;64(6): 974-80

9. Dalela D, Jeong W, Prasad MA, Sood A, Abdollah F, Diaz M, et al. A pragmatic randomized controlled trial examining the impact of the Retziussparing approach on early urinary continence recovery after robot-assisted radical prostatectomy. Eur Urol. 2017;72(5):677-85.

10. Stonier T, Simson N, Davis J, Challacombe B. Retzius-sparing robot-assisted radical prostatectomy (RS-RARP) vs standard RARP: it's time for critical appraisal. BJU Int. 2019;123(1):5-7.

11. Mottet N, Bellmunt J, Bolla M, Briers E, Cumberbatch MG, De Santis M, et al. EAU-ESTRO-SIOG guidelines on prostate Cancer. Part 1: screening, diagnosis, and local treatment with curative intent. Eur Urol. 2017;71(4):618-29.

12. Abdollah F, Dalela D, Sood A, Sammon J, Cho R, Nocera L, et al. Functional outcomes of clinically high-risk prostate cancer patients treated with robotassisted radical prostatectomy: a multi-institutional analysis. Prostate Cancer Prostatic Dis. 2017:20(4):395-400

13. Stewart SB, Boorjian SA. Radical prostatectomy in high-risk and locally advanced prostate cancer: Mayo Clinic perspective. Urol Oncol. 2015;33(5): 235-44.

14. Hager B, Kraywinkel K, Keck B, Katalinic A, Meyer M, Zeissig SR, et al. Increasing use of radical prostatectomy for locally advanced prostate cancer in the USA and Germany: a comparative population-based study. Prostate Cancer Prostatic Dis. 2017:20(1):61-6.

15. Jang TL, Patel N, Faiena I, Radadia KD, Moore DF, Elsamra SE, et al. Comparative effectiveness of radical prostatectomy with adjuvant radiotherapy versus radiotherapy plus androgen deprivation therapy for men with advanced prostate cancer. Cancer. 2018:124(20):4010-22.

16. Dindo D, Demartines N, Clavien PA. Classification of surgical complications: a new proposal with evaluation in a cohort of 6336 patients and results of a survey. Ann Surg. 2004;240(2):205-13.

17. Phukan C, McLean A, Nambiar A, Mukherjee A, Somani B, Krishnamoorthy R, et al. Retzius sparing robotic assisted radical prostatectomy vs. conventional robotic assisted radical prostatectomy: a systematic review and metaanalysis. World J Urol. 2019; https://doi.org/10.1007/s00345-019-02798-4. [Epub ahead of print].

18. Cadeddu JA. Re: a pragmatic randomized controlled trial examining the impact of the Retzius-sparing approach on early urinary continence recovery after robot-assisted radical prostatectomy. J Urol. 2018;199(4): $875-6$. 
19. Yuh B, Artibani W, Heidenreich A, Kimm S, Menon M, Novara G, et al. The role of robot-assisted radical prostatectomy and pelvic lymph node dissection in the management of high-risk prostate cancer: a systematic review. Eur Urol. 2014;65(5):918-27.

20. Choo MS, Kim M, Ku JH, Kwak C, Kim HH, Jeong CW. Extended versus standard pelvic lymph node dissection in radical prostatectomy on oncological and functional outcomes: a systematic review and metaanalysis. Ann Surg Oncol. 2017;24(7):2047-54.

21. Briganti A, Chun FK, Salonia A, Suardi N, Gallina A, Da Pozzo LF, et al. Complications and other surgical outcomes associated with extended pelvic lymphadenectomy in men with localized prostate cancer. Eur Urol. 2006;50(5):1006-13.

22. Saika T, Miura N, Fukumoto T, Yanagihara Y, Miyauchi Y, Kikugawa T. Role of robot-assisted radical prostatectomy in locally advanced prostate cancer. Int J Urol. 2018;25(1):30-5.

23. Harty NJ, Kozinn SI, Canes D, Sorcini A, Moinzadeh A. Comparison of positive surgical margin rates in high risk prostate cancer: open versus minimally invasive radical prostatectomy. Int Braz J Urol. 2013;39(5):639-46 discussion 47-8.

24. Lightfoot AJ, Su YK, Sehgal SS, Lee Z, Greaves GH, Yu SJ, et al. Positive surgical margin trends in patients with pathologic $T 3$ prostate Cancer treated with robot-assisted radical prostatectomy. J Endourol. 2015;29(6): 634-9.

25. Hwang WL, Tendulkar RD, Niemierko A, Agrawal S, Stephans KL, Spratt DE, et al. Comparison between adjuvant and early-salvage Postprostatectomy radiotherapy for prostate Cancer with adverse pathological features. JAMA Oncol. 2018;4(5):e175230.

26. Abdollah F, Sood A, Sammon JD, Hsu L, Beyer B, Moschini M, et al. Longterm cancer control outcomes in patients with clinically high-risk prostate cancer treated with robot-assisted radical prostatectomy: results from a multi-institutional study of 1100 patients. Eur Urol. 2015;68(3):497-505.

27. Gandaglia G, De Lorenzis E, Novara G, Fossati N, De Groote R, Dovey Z, et al. Robot-assisted radical prostatectomy and extended pelvic lymph node dissection in patients with locally-advanced prostate Cancer. Eur Urol. 2017; 71(2):249-56.

28. Michl U, Tennstedt P, Feldmeier L, Mandel P, Oh SJ, Ahyai S, et al. Nervesparing surgery technique, not the preservation of the neurovascular bundles, leads to improved long-term continence rates after radical prostatectomy. Eur Urol. 2016;69(4):584-9.

29. Seikkula H, Janssen P, Tutolo M, Tosco L, Battaglia A, Moris L, et al. Comparison of functional outcome after extended versus super-extended pelvic lymph node dissection during radical prostatectomy in high-risk localized prostate Cancer. Front Oncol. 2017;7:280.

30. Adam M, Tennstedt P, Lanwehr D, Tilki D, Steuber T, Beyer B, et al. Functional outcomes and quality of life after radical prostatectomy only versus a combination of prostatectomy with radiation and hormonal therapy. Eur Urol. 2017;71(3):330-6.

31. Nyarangi-Dix JN, Steimer J, Bruckner T, Jakobi H, Koerber SA, Hadaschik B, et al. Post-prostatectomy radiotherapy adversely affects urinary continence irrespective of radiotherapy regime. World J Urol. 2017;35(12):1841-7.

32. Chang LW, Hung SC, Hu JC, Chiu KY. Retzius-sparing robotic-assisted radical prostatectomy associated with less bladder neck descent and better early continence outcome. Anticancer Res. 2018:38(1):345-51.

33. Imbimbo C, Creta M, Gacci M, Simonato A, Gontero P, de Cobelli O, et al. Patients' desire to preserve sexual activity and final decision for a nervesparing approach: results from the MIRROR (multicenter Italian report on radical prostatectomy outcomes and research) study. J Sex Med. 2011;8(5): 1495-502.

\section{Publisher's Note}

Springer Nature remains neutral with regard to jurisdictional claims in published maps and institutional affiliations.

Ready to submit your research? Choose BMC and benefit from:

- fast, convenient online submission

- thorough peer review by experienced researchers in your field

- rapid publication on acceptance

- support for research data, including large and complex data types

- gold Open Access which fosters wider collaboration and increased citations

- maximum visibility for your research: over $100 \mathrm{M}$ website views per year

At BMC, research is always in progress.

Learn more biomedcentral.com/submissions 